\title{
PARAMETRIC EXTRAPOLATION AS A PARALLEL METHOD IN MATHEMATICAL PHYSICS
}

\author{
DANG QUANG A
}

\begin{abstract}
In recent years we have developed a parallel method for mathematical physics problems. It is the method of parametric extrapolation. In this paper we give an overview of our results concerning this method for constructing parallel algorithms for some problems of mathematical physics.
\end{abstract}

Tóm tăt. Trong nhũng năm gần đây chúng tôi đã phát triễn một phương pháp song song giải một số bài toán biên cưa vật lý - toán. Đó là phưong pháp ngoại suy theo tham số. Bài báo này là tỗng quan các kết quả nghiên cứu cưa chúng tôi liên quan đến phưong pháp này để xây dựng các thuật toán song song giải một số bài toán biên cho phương trình elliptic cấp hai và cấp bốn ờ múc vi phân cũng như ơ mức rời rạc.

\section{INTRODUCTION}

Now, coping with large-scale problems of physics, mechanics, oceanology, meteorology, hydrology,... one has to use parallel computing systems in order to reduce computation time. For this reason it should construct paralell methods and algorithms for the problems to be realized on the parallel systems. For the parallel solution of boundary value problems (BVPs) for partial differential equations three main directions can be distinguished: approaches based on "parallelism across the problem", "parallelism across method" and on "parallelism across steps". Among the directions, the second approach of method-parallelism received much attention. Here it is worth to mention the domain decomposition methods and the parallel splitting up methods. In recent years we have developed an another parallel method for mathematical physics problems. It is the method of parametric extrapolation. In this paper we give an overview of our results concerning this method for constructing parallel algorithms for some problems of mathematical physics.

\section{THE IDEA OF THE METHOD}

\subsection{From the method of parametric correction of difference schemes...}

The idea of the method is originated from the method of parametric correction of difference schemes proposed by Belotserkovskij and his colleagues [3] in 1984. Their goal then was to solve the conflict between the stability and high order approximation of difference schemes for hyperbolic problems and to increase the effectiveness of iterative processes for second order elliptic problems. In order to do this for each BVP they constructed a manifold of difference schemes depending on two or more parameters instead of one as it was usually done before. Due to this manifold of difference schemes they could get new properties of the difference scheme which is a appropiate linear combination of basic difference schemes. Speaking roughly, the idea of the method of parametric correction of difference schemes is that a "good" difference scheme may be obtained in the result of combining "bad" ones by the suitable selection of parameters. The realization of this method leads to the concept of the generalized difference scheme as a combination of the basic difference schemes with some weights, which was discussed in [4] and applied for studying discontinuous solutions of the wave equation in [5]. The results of computation in the latter paper allows to conclude that the consideration of a family of difference schemes constructed by special way not only opens a possibility

\footnotetext{
* This work is supported by the National Basic Research Program in Natural Sciences.
}

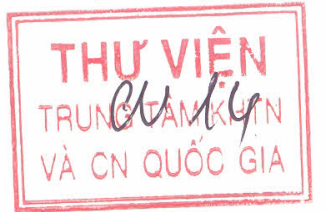


to increase the effectiveness of difference scheme but also reaches more adequacy of discrete model to the phenomena studied. Indeed, it is proposed to construct the discrete model of continuous media from several discrete models, each of those is not adequated to the continuous model. But the difference between the discrete models is organized so that they may be controlled. The family of these models due to their constructive character may be made rational and when being considered as a new model can possess new properties which each separate model does not have. For this reason the method of parametric correction of difference schemes is considered as a new principle in the construction of discrete models in mechanics of continuous media.

The method of parametric correction of difference schemes were used by ourselves in [7] for constructing generalized difference schemes quasimonotone and having high order of accuracy for some equations and systems. It is in the latter paper, the conflict between the stability and high order approximation solved not completely in [3] was solved fully. But the problem, in which we are interested most, is the construction of efficient iterative methods for solving BVPs for elliptic equations on both differential and difference levels.

\section{2. ... To the parameter extrapolation method}

Below we present the idea of the parameter extrapolation method for a general equation.

Let $A$ be a linear symmetric, positive definite operator in a Hilbert space $H$. Consider the equation

$$
A u=f, \quad f \in R(A)
$$

This equation may be solved by known iterative methods with the rate of convergence depending on the ratio $M / m$. Here $M$ and $m$ are maximal and minimal eigenvalues of the operator $A$, respectively. In the case, where $H$ is of infinite dimension and 0 is the limit point of the spectrum of the operator, in general one has not obtained or obtained very bad results of the convergence rate of the methods. In order to overcome this difficulty, and also to increase the convergence rate of the iterative processes, we propose instead (1) to solve some perturbed problems

$$
(A+\varepsilon P) u_{\varepsilon}=f
$$

where $P$ is a linear symmetric, positive definite operator suitably selected for every specified operator $A$. Then, we extrapolate by the parameter $\varepsilon$ the solutions of (2), i.e., take the combination

$$
U^{e}=\sum_{k=1}^{N+1} \gamma_{k} u_{\varepsilon / k}
$$

with $\gamma_{k}$ chosen as follows

$$
\gamma_{k}=\frac{(-1)^{N+1-k} k^{N+1}}{k !(N+1-k) !}
$$

be an approximate solution of (1). For the error of the approximate solution we have the estimate

$$
\left\|U^{e}-u^{*}\right\| \leq C \varepsilon^{N+1}
$$

This result is obtained with the help of the expansion

$$
u_{\varepsilon}=u^{*}+\sum_{k=1}^{N} \varepsilon^{k} v_{k}+\varepsilon^{N+1} w_{\varepsilon}
$$

where $u^{*}$ is the solution of the original equation (1), $v_{k}$ are elements of $H$ independent of $\varepsilon, w_{\varepsilon}$ is uniformly bounded in $\varepsilon, N$ is an integer depending on $A$.

The mentioned above fact is proved in [10].

Thus, the direct solving of (1) is replaced by solving $N$ perturbed problems (2) with the parameters $\varepsilon / k, \quad(k=1, \ldots, N)$. These problems may be solved simultaneously on processors of parallel computers. The advantage of this method is that known iterative methods applied directly to (1) 
are slowly converged, even may be, are deverged, while known iterative methods applied to (2) will converge fastly with the rate of geometric progression.

Comment 1 (Tikhonov regularization). The equation (2) in some sense is the Tikhonov regularized equation for (1) (for Tikhonov regularization see e.g. [28]). Here we extrapolate its solution depending on the regularization parameter for obtaining the solution of the original equation (1).

Comment 2 (Richardson extrapolation). In the proposed method, the extrapolation is perfomed by a small parameter introduced into the original equation in order to make some perturbation. Differently from this, the well-known Richardson extrapolation (see, e.g. [23]) is by the stepsize of discretization of differential problem. Due to this extrapolation the order of accuracy of difference scheme is increased. It is possible to be realized with the help of the asymptotic error expansions to finite difference schemes.

In the following sections we shall summarize results of using the method of parametric extrapolation for some problems on differential and difference levels. It should be noticed that for differential problems, in order to apply this method, the most important step is the reduction of the problems under consideration to an equation with a symmetric, positive definite and completely continuous in a Hilbert space. Therefore, in Sections 2 and 9 we only sketch how to reduce original BVPs to corresponding operator equations in Hilbert. space.

\section{THE DIRICHLET PROBLEM FOR SECOND ORDER ELLIPTIC EQUATION WITH DISCONTINUOUS COEFFICIENTS}

Let $\Omega$ be a bounded domain in the m-dimensional Eucledean space $R^{m}$ with Lipshitz boundary $S$. Denote by $\Omega_{+}$a proper subdomain of $\Omega$ with boundary $\Gamma, \nu$ the outward normal to $\Gamma$.

Consider the boundary value problem (BVP)

$$
\begin{aligned}
L u \equiv \sum_{i, j=1}^{m} \frac{\partial}{\partial x_{i}}\left(a_{i j}(x) \frac{\partial u}{\partial x_{j}}\right) & =f(x), \quad x \in \Omega \backslash \Gamma, \\
a(x) & \geq \delta>0, \\
{[u]_{\Gamma}=0, \quad\left[\frac{\partial u}{\partial \nu}\right]_{\Gamma} } & =0,\left.\quad u\right|_{S}=\Phi,
\end{aligned}
$$

where $\left[\left.u\right|_{\Gamma}\right.$ is the jump of $u$ through $\Gamma:[u]_{\Gamma}=u^{+}-u^{-}, \quad u^{ \pm}(x)=u(x), \quad x \in \Omega_{ \pm}, \quad \frac{\partial u^{ \pm}}{\partial \nu_{ \pm}}$are conormal derivatives of $u^{ \pm}$.

By the introduction of a boundary operator $K$, defined as follows

$$
K: \quad g \rightarrow|w|_{\Gamma},
$$

where $g$ is a boundary function defined on $\Gamma, w$ is the solution of the problem

$$
\begin{aligned}
& L u=0, \quad x \in \Omega \backslash \Gamma,\left.\quad w\right|_{S}=0, \\
& \left.\frac{\partial w^{+}}{\partial \nu+}\right|_{\Gamma}=g, \quad\left[\frac{\partial w}{\partial \nu}\right]_{\Gamma}=0,
\end{aligned}
$$

the problem (4) is reduced to the operator equation

$$
K g=F,
$$

here $F$ is a function depending linearly on $f$ and $\Phi$. There was proved that $K$ is a linear, symmetric, positive definite and completely continuous in the space $L_{2}(\Gamma)$. Instead of solving (6), we consider perturbed equation

$$
(K+\varepsilon I) g_{\varepsilon}=F
$$

where $I$ is the identity operator. 
This equation is lead from the perturbed problem

$$
\begin{gathered}
L u_{\varepsilon}=f(x), x \in \Omega \backslash \Gamma,\left.\quad u_{\varepsilon}\right|_{S}=\Phi, \\
\left.\varepsilon \frac{\partial u_{\varepsilon}^{+}}{\partial \nu_{+}}\right|_{\Gamma}+\left[u_{\varepsilon}\right]_{\Gamma}=0, \quad\left[\frac{\partial u_{\varepsilon}}{\partial \nu}\right]_{\Gamma}=0,
\end{gathered}
$$

The simple iterative method applied to (7) is converged with the rate of geometric progression, while the iterative method of Osmolovskij \& Rivkind [24] for the original problem (4) only is convereged with the rate $O\left(1 / N^{\alpha}\right)$, where $N$ is the number of iterations, $\alpha$ is a number depending on the smoothness of the solution. It is interesting that the realization of the iterative method for (6) and for (7) leads to the successive solution of a sequence of BVP in each of the subdomains, where the Neumann condition on the interface is step by step made more precise.

Comment 3 (domain decomposition methods). The proposed above method is applicable for the problem where the domain $\Omega$ consists of two subdomains which except for the interface have their proper boundary. Thus, the approximate solution is constructed by the extrapolation of the solutions found by a domain decomposition method. It should be notice that at present domain decomposition methods attract great attention from many researchers (see c.f. $[2,21,22,26,29]$ ) due to the needs to solve BVPs in geometrically complicated domains. Besides the way of making the Neumann boundary values more precise on the interface as in our work [12], many other authors proposed to do so with the Dirichlet boundary values or alternatively exchange the Neumann and Dirichlet boundary values.

Comment 4 (boundary element methods). After reducing the original and the associated perturbed problems to boundary operator equations we don't intend to solve them by numerical methods, for example, boundary element methods, but only use them as means for studing the convergence of iterative process for BVPs.

\section{BVPS FOR BIHARMONIC, BIHARMONIC TYPE AND TRIHARMONIC EQUATIONS}

4.1. Solving BVPs for the fourth order differential equation by the reduction of them to BVPs for the second order equations with the aim to use a lot of efficient algorithms for the latter ones attracts attention from many researchers. Namely, for the biharmonic equation $\Delta^{2} u=f$ with the Dirichlet boundary condition, there is intensively developed the iterative method, which leads the problem to two problems for the Poisson equation at each iteration (see e.g. $[20,25]$ ). But unfortunately, in these works the convergence rate of the iterative process either was not obtained [20] or is very low, namely, is of order $O(1 / N)$, where $N$ is the number of iterations [25]. In order to elaborate faster algorithms for the biharmonic equation, in [8] first time we applied the parameter extrapolation technique to this equation. For reducing the Dirichlet problem for the biharmonic equation to a boundary operator equation we defined the boundary operator via Green functions as was done in [6]. The result of computation implemented in [9] confirmed the advantage of the parametric extrapolation technique.

4.2. The technique for reducing BVP for biharmonic equation to boundary operator equation in the mentioned above papers is improved in our further works when being applied to a mixed BVP for the biharmonic equation [16] and for BVPs for biharmonic type equation [13-15]. Below we briefly demonstrate this technique for the Dirichlet problem

$$
\begin{aligned}
& L u \equiv \Delta^{2} u-a \Delta u+b u=f(x), x \in \Omega, \\
& \left.u\right|_{\Gamma}=u_{0},\left.\frac{\partial u}{\partial \nu}\right|_{\Gamma}=u_{\nu} .
\end{aligned}
$$

Here $\Omega$ is a bounded domain in $R^{m}, \Delta$ is the Laplace operator, $a \geq 0, b \geq 0$.

4.2.1. Suppose that $a>0$ and

$$
a^{2}-4 b \geq 0,
$$


We introduce boundary operator $B$ by the formula

$$
B v_{0}=\left.\frac{\partial u}{\partial \nu}\right|_{\Gamma}
$$

where $v_{0}$ is a function defined on $\Gamma, u$ solves the problems

$$
\begin{array}{ll}
L_{2} v=0, & x \in \Omega,\left.\quad v\right|_{\Gamma}=v_{0}, \\
L_{1} u=v, & x \in \Omega,\left.\quad u\right|_{\Gamma}=0 .
\end{array}
$$

Here $L_{1}, L_{2}$ are the factors in the factorization of $L$, whose formulae are given in [13]. Then the problem (9) is reduced to the operator equation

$$
B v_{0}=F,
$$

with $B=B^{*}>0$ and completely continuous in $L_{2}(\Gamma)$, linearly expressing through $u_{0}, u_{\nu}, f$. Rather than (11) we solve the perturbed equation

$$
(B+\delta I) v_{\delta 0}=F, \quad \delta>0
$$

This equation is obtained from the perturbed problems

$$
\begin{gathered}
L u_{\delta} \equiv \Delta^{2} u_{\delta}-a \Delta u_{\delta}+b u_{\delta}=f(x), x \in \Omega, \\
\left.u_{\delta}\right|_{\Gamma}=u_{0},\left.\delta\left(\frac{1}{\mu} \Delta u_{\delta}-u_{\delta}\right)\right|_{\Gamma}+\left.\frac{\partial u_{\delta}}{\partial \nu}\right|_{\Gamma}=u_{\nu}
\end{gathered}
$$

where $\mu=\frac{1}{2}\left(a+\sqrt{a^{2}-4 b}\right)$.

It should be emphasized that the simple iteratation method for the equation (12) is convergent with the rate of geometric progression and is realized by solving a sequence of BVPs for second order equations, while the iterative method for the biharmonic type equation (9) in [1] is not proved to be convergent.

4.2.2. Now consider the case, where and the condition (10) is not satisfied. For brief we set $u_{0}=$ $u_{\nu}=0$.

We introduce a mixed domain-boundary operator $B$, defined by the formula $B: w \rightarrow B w$, where

$$
w=\left(\begin{array}{c}
v_{0} \\
D
\end{array}\right), \quad B w=\left(\begin{array}{c}
\left.b \frac{\partial u}{\partial \nu}\right|_{\Gamma} \\
D+b u
\end{array}\right),
$$

$u$ is the function found from the problems

$$
\begin{aligned}
\Delta v-a v & =D, x \in \Omega,\left.\quad v\right|_{\Gamma}=v_{0}, \\
\Delta u & =v, x \in \Omega,\left.\quad u\right|_{\Gamma}=0 .
\end{aligned}
$$

It was proved that $B=B^{*}>0$ and $B$ is bounded in the space $L_{2}(\Gamma) \times L_{2}(\Omega)$ and has expansion $B=B_{0}+I_{2}$, where $B_{0}=B_{0}^{*}>0$ is completely continuous, $I_{2}$ is a projector on $L_{2}(\Omega)$, namely, $I_{2} w=\left(\begin{array}{c}0 \\ D\end{array}\right)$.

Then the BVP is reduced to the operator equation

$$
B w=F,
$$

here for brevity we omit the concrete expression of $F$. If apply any iterative method immediately, for example, the two-layer iterative scheme to (13) then we can not say anything about its convergence. Hence, instead of (13) we consider the equation

$$
\left(B+\delta I_{1}\right) w_{\delta 0}=F, \quad, 0<\delta<1
$$

where $I_{1}$ is a projector on $L_{2}(\Gamma)$, i.e. $I_{1} w=\left(\begin{array}{c}v_{0} \\ 0\end{array}\right)$. 
We have $B+\delta I_{1} \geq B_{0}+\delta I \geq \delta I$ Consequently, two-layer iterative scheme for (14) will be convergent with the rate of geometric progression.

The perturbed problem (14) is lead from the original problem, where the boundary condition $\left.\frac{\partial u}{\partial \nu}\right|_{\Gamma}=0$ is replaced by $\left.\left(\delta \Delta u+\frac{\partial u}{\partial \nu}\right)\right|_{\Gamma}=0$.

4.3. The technique for reducing BVP to boundary operator equation in order to apply the method of parametric extrapolation recently found a new application [18] for the following triharmonic problem

$$
\begin{aligned}
\Delta^{3} u & =f(x), \quad x \in \Omega, \\
\left.u\right|_{\Gamma} & =0,\left.\quad \frac{\partial u}{\partial \nu}\right|_{\Gamma}=0,\left.\quad \Delta u\right|_{\Gamma}=0 .
\end{aligned}
$$

For this problem we introduce a boundary operator $B$ defined on boundary functions by the formula

$$
B w_{0}=-\left.\frac{\partial u}{\partial \nu}\right|_{\Gamma},
$$

where $w_{0}$ is a function defined on $\Gamma$ and $u$ solves the problems

$$
\begin{array}{rlrl}
\Delta w & =0, & x \in \Omega, & \left.w\right|_{\Gamma}=w_{0}, \\
\Delta v & =w, \quad x \in \Omega, & \left.v\right|_{\Gamma}=0, \\
\Delta u & =v, \quad x \in \Omega, & \left.u\right|_{\Gamma}=0 .
\end{array}
$$

Then the problem (15) is reduced to the operator equation

$$
B w_{0}=F,
$$

with $B=B^{*}>0$ and completely continuous in $L_{2}(\Gamma), F$ linearly expressing through $f$. The perturbed equation of (15) is obtained from the corresponding perturbed BVP

$$
\begin{aligned}
\Delta^{3} u_{\delta} & =f(x), \quad x \in \Omega, \\
\left.u_{\delta}\right|_{\Gamma} & =0,\left.\quad \Delta u_{\delta}\right|_{\Gamma}=0,\left.\quad\left(\frac{\partial u_{\delta}}{\partial \nu}-\delta \Delta^{2} u_{\delta}\right)\right|_{\Gamma}=0,
\end{aligned}
$$

and the realization of the iterative method for it leads to the solution of three Dirichlet problems for the Poisson equation.

Comment 5 (perturbation of boundary condition). The essential difference from the method of parametric correction of difference schemes $[3-5,7]$, where the difference operator approximating a differential equation is made perturbed, is that in our works $[13,14-16,18]$ we consider a family of BVPs with one perturbed boundary condition. Hence, after the reduction of them to boundary operator equation we obtained a family of boundary operator equations depending on a parameter and the extrapolation is performed by this parameter.

\section{ACCELERATING THE CONVERGENCE RATE OF ITERATIVE METHODS FOR SOLVING GRID EQUATIONS AND DEGENERATE SYSTEM OF ALGEBRAIC EQUATIONS}

5.1. The design of fast algorithms for large-scale systems of linear algebraic equations is a very actual problem attracting great attention from both mathematicians and engineers. These large systems usually arise in the result of discretization of BVPs for two- or three-dimensional elliptic equations on thin grids. There are a lot of works concerning this problem (see e.g. monographs [22, 27] and references therein). In $[10,11,17]$ we proposed to use the method of parametric extrapolation for accelerating the convergence rate of well-known iterative methods. The matter is as follows. 
Consider the operator equation

$$
A u=f
$$

in the $N$-dimensional Eucledean space with $A=A^{*} \geq \delta I, \delta>0$. For solving this equation one usually constructs two-layer iterative schemes of the form [27]

$$
B \frac{y_{k+1}-y_{k}}{\tau_{k+1}}+A y_{k}=f, \quad k=0,1, \ldots
$$

where $B$ is easily invertable operator and is energetic equivalent to $A$ :

$$
\gamma_{1} B \leq A \leq \gamma_{2} B, \quad \gamma_{2} \geq \gamma_{1}>0 .
$$

Then the rate of convergence of (15) depends on the ratio $\xi=\gamma_{1} / \gamma_{2}$

For this purpose one starts from an operator $R=R^{*}>0$ energetic equivalent to $A$ :

$$
c_{1} R \leq A \leq c_{2} R, \quad c_{2} \geq c_{1}>0 .
$$

For accelerating the iterative process we propose to solve (2) rather than (1a) with $P$ chosen specifically as follows:

Case 1. If $R=R_{1}+R_{2}, R_{2}^{*}=R_{1}, R_{1} R_{2} \neq R_{2} R_{1}$ we choose $P=R_{1} R_{2}$ and apply the alternating triangles method to (2).

Case 2. If $R=R_{1}+R_{2}, R_{1}^{*}=R_{1}, R_{2}^{*}=R_{2}, R_{1} R_{2}=R_{2} R_{1}$ we choose $P=R_{1} R_{2}$ and apply the alternating directions method to (2).

Case 3. If $R=R_{1}+R_{2}+R_{3}, R_{i}^{*}=R_{i}, R_{i} R_{j}=R_{j} R_{i},: i, j=1,2,3$ we choose $P=R_{1} R_{2}+$ $R_{2} R_{3}+R_{1} R_{3}+\sqrt{h} R_{1} R_{2} R_{3}$, where $h$ is the grid step for discretization of differential problems, and then apply the alternating directions method to (2). The detailed proof and examples illustrating the effectiveness of the proposed method are presented in papers $[10,11]$.

In the case, where $A$ is degenerate operator, restricting ourselfes in the image of $A,(\operatorname{Im} A)$, we also obtained analogous results concerning iterative processes for the normal solution of (1a) (see $[17])$.

Comment 6. The use of the method of parametric extrapolation does not exclude the possibility of using other fast methods for systems of linear algebraic equations. Moreover, its efficiency will be increased if combine it with one of the fast methods for the perturbed system (2).

5.2. For finding the normal solution of a system of linear algebraic equations (1a) with symmetric, nonnegative degenerate matrix $A$ in the case of consistency some authors used a simplified Tikhonov regularization method, namely, the method of shifting spectrum, i.e. they consider the system

$$
(A+\alpha I) u_{\alpha}=f .
$$

This system has a unique solution and when $\alpha$ tends to zero this solution approaches to the normal solution. But the problem of estimating computational work for obtaining an approximate solution with a given accuracy has not been considered yet. Theoretically, in order to obtain a good approximation of the normal solution we must choose $\alpha$ small enough. But when it is very small then the matrix $A$ is ill-conditioned. Therefore, if the size of the system is large enough, for solving the system we should use iterative methods and then the iterative methods converge very slowly. It implies that for obtaining an approximate solution to the normal solution with a given accuracy a very great computational work should be carried out. In the case if the size of the system is so small that direct methods for (23) can be applied the experiments show that when $\alpha$ is less than a threshold the result of computation is oscillating. In order to achieve the normal solution of the system (1a) with a given accuracy $\varepsilon$ spending the possibly minimal computational cost we propose to use the method of extrapolation by the regularization parameter $\alpha$ (see [19]). We have obtained the following estimate

$$
\frac{\left\|U^{E}-u^{*}\right\|}{\left\|u^{*}\right\|} \leq \frac{\alpha^{k+1}}{\lambda_{\min }^{k+1}},
$$


where $u *$ is the normal solution, $U^{e}$ is the extrapolated solution by $k+1$ solutions of $(18), \lambda_{m}$ in is the smallest eigenvalue of $A$. From this estimate we establish that if applying the simple iterative method $[22,27]$ to $(18)$ then for achieving the normal solution of (1a) with the given accuracy $\varepsilon$ by using the parametric extrapolation we reduce the computational amount $G$ times in comparison with using only one shifted equation (18),

$$
G=\left[\frac{2}{(k+1)(k+2) \varepsilon^{k /(k+1)}}\right] .
$$

In the case if the system (1a) is inconsistent then the solution of (18) does not approximate the normal solution. Nevertheless, we proved that extrapolating $k+1$ solutions of (18) with parameters $\alpha / j(j=1, \ldots, k+1)$ we get an approximation of the normal solution with the estimate

$$
\frac{\left\|U^{e}-u^{*}\right\|}{\left\|u^{*}\right\|} \leq \frac{\alpha^{k}}{\lambda_{\min }^{k}}
$$

Remark. In the case when the matrix $\mathrm{A}$ is not symmetric the Tikhonov regularization leads to the solution of the system

$$
\left(A^{*} A+\alpha I\right) u_{\alpha}=A^{*} f
$$

Using the extrapolation by the parameter $\alpha$ we obtained the same result as (19) for both consistent and inconsistent systems (1a).

\section{CONCLUDING REMARK}

The major work in the realization of the method of parametric extrapolation is the parallel solution of the perturbed problem with some various values of the parameter, each on a processor. The computation of the extrapolated solution as a combination of the perturbed solutions is only the last simple work. Thus, the degree of parallelization of the method is very high.

Acknowledgement. We wish to thank an anonymous referee for his valuable comments and suggestions which improved the paper.

\section{REFERENCES}

[1] Abramov A. A. and Ulijanova V.I., On a method for solving biharmonic type equation with singularly small parameter, J. of Comp. Math. and Math. Phys. 32(4) (1992) 567-575 (Russian).

[2] Agoshkov V.I., Lebedev B. I., Poincare-Steklov operators and domain decomposition methods in variational problems, In book: Computing Processes and Systems, Issue 2, Nauka, Moscow, 1985, 173-227 (Russian).

[3] Belotserkovskij O. M., Panarin A. I., Tshennikov V.V., Method of parameter correction of difference schemes, J. of Comp. Math. and Math. Phys. 24 (1) (1984) 65-74 (Russian).

[4] Belotserkovskij O. M., Panarin A. I., Tshennikov V.V, Generalized difference schemes and the method of parameter correction of difference schemes, In book: Cybernetics and Computing Technics, Issue 1, Moscow, 1986, 99-117 (Russian).

[5] Belotserkovskij O. M., Panarin A. I., Tshennikov V.V., Discontinuous solutions and generalized difference schemes, In book: Cybernetics and Computing Technics, Issue 2, Moscow, 1986, 95104 (Russian).

[6] Dang Quang A, On an iterative method for a boundary value problem for a fourth order differential equation, Math. Phys. and Nonlin. Mech. 44 (10) (1988) 54-59 (Russian).

[7] Dang Quang A, Construction of quasimonotone generalized difference schemes having high order of accuracy for some equations, J. of Comp. Sci. and Cyber. 6 (2) (1990) 15-20 (Vietnamese).

[8] Dang Quang A, Application of extrapolation to constructing effective method for solving the Dirichlet problem for biharmonic equation, Institute of Computer Science, Preprint No. 5, 1990. 
[9] Dang Quang A, Numerical method for solving the Dirichlet problem for fourth order differential equation, Proc. of the Srd National Conference on Gas and Fluid Mechanics, Hanoi, 1991, 195199 (Vietnamese).

[10] Dang Quang A, Accelerated method for solving grid equations, I, J. of Comp. Sci. and Cyber. 9 (3) (1993) 22-32.

[11] Dang Quang A, Accelerated method for solving grid equations, II (3-D case), J. of Comp. Sci. and Cyber. 11 (2) (1995) 1-6.

[12] Dang Quang A, Approximate method for solving an elliptic problem with discontinuous coefficients, Journal of Comput. and Applied Math. 51 (2) (1994) 193-203.

[13] Dang Quang A, Boundary operator method for approximate solution of biharmonic type equation, Vietnam Journal of Math. 22 (1\&2 (1994) 114-120.

[14] Dang Quang A, Iterative method for solving the second boundary value problem for biharmonic type equation, J. of Comp. Sci. and Cyber. 14 (4) (1998) 66-72.

[15] Dang Quang A, Mixed boundary-domain operator in approximate solution of biharmonic type equation, Victnam Journal of Math. 26 (3) (1998) 249-258.

[16] Dang Quang A, Construction of iterative methods for solving a mixed boundary value problem for biharmonic equation, Proceedings of the fifth Mathematical Conference of Vietnam, Hanoi, September 17-20, 1997, Sci. and Tech. Publ. House, Hanoi, 1999, 47-55.

[17] Dang Quang A, Iterative methods for solving degenerate system of grid equations, J. of Comp. Sci. and Cyber. 13 (4) (1997) 33-45.

[18] Dang Quang A, Iterative method for solving a boundary value problem for triharmonic equation, Vietnam Journal of Math. (accepted).

[19] Dang Quang A, Parametric extrapolation method for degenerate system of linear algebraic equations (submitted).

[20] Glowinski R., Lions J. L., and Tremoliere, Analyse Numerique des Inequations Variotionelles, Dunod, Paris, 1976.

[21] Glowinski R., Golub G.H., and Periaux J, Eds, Proc. First International Symposium on Domain Decompostion Methods, SIAM, Philadelphia, 1988.

[22] Marchuk G.I., Methods of Numerical Mathematics, Nauka, Moscow, 1989 (Russian).

[23] Marchuk G.I., Shaidurov V. V., Difference Methods and Extrapolations, Springer, Berlin, 1983.

[24] Osmolovskij V.G. and Rivkind V. Ya, Domain decomposition method for the second order elliptic equation with discontinuous coefficients, J. of Comp. Math. and Math. Phys. 21 (1) (1981) 33-38 (Russian).

[25] Palsev B. V., On the expansion of the Dirichlet problem and a mixed problem for biharmonic equation into a series of decomposed problems, J. of Comp. Math. and Math. Phys. 6 (1) (1966) 43-51 (Russian).

[26] Rice J.R., Vavalis E.A., Yang Daoqi, Analysis of a nonoverlapping domain decomposition method for elliptic partial differential equations, Journal of Comput. and Applied Math. 87 (1997) 11-19.

[27] Samarskij A. and Nikolaev E., Numerical Methods for Grid Equations, Vol. 2, Birkhauser, Basel, 1989.

[28] Tikhonov A. N., Goncharsky A.V., Stepanov V.V., and Yagola A. G., Numerical Methods for the Solution of Ill-Posed Problems, Dordrecht - Kluwer Acad. Publ., 1995.

[29] Zhu Jialin, Domain decomposition methods with boundary elements, In book: Boundary Element Methods, Proc. of the Fifth Japan-China Symposium on Boundary Element Methods, Elsevier, Amsterdam - London - New York - Tokyo, 1993, 61-66.

Received December 14, 1999 Revised January 30, 2001 